\title{
A INFLUÊNCIA DAS PRIORIDADES COMPETITIVAS NA GESTÃO GLOBAL DA CADEIA DE SUPRIMENTO - UM ESTUDO MULTICASO
}

Alexandre Luis Prim ${ }^{1}$ Jeferson Deleon Fávero ${ }^{2}$

\section{RESUMO}

A globalização influenciou o crescimento competitivo, rompendo barreiras e proporcionando maiores desafios nas organizações. A adoção de novas prioridades competitivas foi necessária para obter vantagem nesse âmbito. $\mathrm{O}$ objetivo desta pesquisa é identificar o grau de importância e o impacto de prioridades competitivas pela gestão global da cadeia de suprimento na busca por vantagens dessa ordem, a qual é demonstrada na hipótese desta pesquisa. A abordagem metodológica se denomina quantitativa explicativa, com estudo multicaso em três organizações multinacionais do segmento metal-mecânico, situadas no Estado de Santa Catarina. Como resultado, identificou-se a confirmação da hipótese: as prioridades de custo, integração, velocidade, flexibilidade, qualidade e preço podem influenciar no desempenho da gestão global da cadeia de suprimento; e a integração, o compartilhamento e a cooperação de informações na cadeia de suprimento condicionam um melhor desempenho no sistema de valor.

Palavras-Chave: TI - Prioridade Competitiva. Gestão Global da cadeia de suprimento. Vantagem competitiva. 


\section{INTRODUÇÃO}

Nas últimas décadas, notoriamente tem-se observado transformações intensas nos aspectos sociais, culturais, políticos e, principalmente, econômicos. (MOTTA, 1995; PESSANHA et al., 2012). Abre-se, no período pós-guerra, um novo desenvolvimento docapitalismo sem limites geográficos, com a integração de nações em nível global - a globalização. (BALANCO, 2008).

A globalização contribuiu enormemente para o acesso de produtos e serviços, porém despertou a competitividade a nível global, aumentando a rivalidade entre empresas e a incerteza do mercado. (JANSEN; ROTONDARO; JANSEN, 2005; HOSKISSON et al., 2009).

A partir desse aspecto, a competitividade entre organizações cresceu exponencialmente, na qual a busca pela vantagem competitiva se tornou fundamental para a sobrevivência e destaque das organizações. (PORTER, 1989). A ênfase se volta à busca inequívoca por formular e implementar estratégias concorrenciais a fim de obter uma posição sustentada no mercado. (FERRAZ; KUPFER; HAGUERNAUER, 1995).

Neste momento, a competitividade extrapola os limites internos de uma organização, caracterizado por Porter (1989) como cadeia de valor, e se volta ao enfoque de um sistema de valor, identificado pelo mesmo autor como envolvido em todos os níveis da cadeia; ou seja, na interação, desde os fornecedores até o consumidor final. Contudo, ocorre, ainda, a mudança do comportamento das organizações, devido à transição de concorrência entre empresas para redes de suprimentos. (HULT; KETCHEN; SLATER, 2004; LI et al., 2005; KOH et al., 2007; CHOW et al., 2008; CORRÊA, 2010; ERTURGUT, 2012).
Com essa influência, a logística passa de um enfoque de execução de serviços de transporte e armazenamento para a incorporação de uma direção estratégica, na concepção de reduzir custos, satisfazer o consumidor, entregar no local e no tempo desejado a um preço justo. (BERTAGLIA,2009;BOWERSOX; CLOSS, 2010; CORREA, 2010).

Para Porter (1989), a vantagem competitiva está na competência das organizações em administrar todo o sistema de valor. No entanto, com o decorrer da dinâmica de mercado, por meio de interações entre o sistema de valor, ocorrem escolhas por prioridades competitivas que conduzam as organizações para obtenção de um melhor desempenho.

NesSA PERSPECTIVA, ESTA PES-

QUISA TEM POR OBJETIVO PRO-

POR UM MODELO OPERACIONAL,

MENSURAR SUA VERACIDADE,

BEM COMO RESPONDER ÀS PER-

GUNTAS DE PESQUISA: EXISTE

INFlUÊNCIA DAS PRIORIDADES

COMPETITIVAS NO DESEMPE-

NHO DA GESTÃO GLOBAL DA

CADEIA DE SUPRIMENTO? QUAIS

SÃO MAIS IMPORTANTES E

INflUENCIADORAS?

A fim de responder tais perguntas de pesquisa e testar o modelo operacional, realizou-se um estudo quantitativo em três organizações multinacionais do segmento metal-mecânico, situadas no Estado de Santa Catarina, Brasil. 


\section{GLOBALIZAÇÃO}

A globalização pode ser definida como a interdependência econômica entre nações, que se reflete no fluxo de bens e serviços, no capital financeiro e no conhecimento que atravessa fronteiras nacionais. (HOSKISSON et al., 2009). Os seus efeitos proporcionam diversos benefícios a todas as nações, embora direcionem uma nova dinâmica econômica às organizações pela necessidade de tornarem-se competitivas de forma sustentada. (OBADIA; VIDAL; MELO,2007). A globalização contribuiu, ainda, para a alta concorrência, a rivalidade acirrada entre empresas e a incerteza do mercado em nível mundial. (JANSEN; ROTONDARO; JANSEN, 2005; HOSKISSON et al., 2009).

Há organizações que têm êxito e outras que fracassam na competição internacional. (PORTER, 1989). Entretanto, para se manter no mercado global, há a necessidade de melhorar e inovar (PORTER, 1999), adotando meios de competição que sustentem o seu posicionamento.

Diante dessa realidade, os avanços tecnológicos são fontes de vantagem competitiva que respondem a essa sustentação. Guido e Lima (2012) destacam, por essa via, a aceleração de processos, rompimento de fronteiras e modificação da forma de relacionamento entre empresas e nações. Nesse enfoque, Hoskisson et al. (2009) relatam que os avanços tecnológicos no cenário competitivo ocorrem com mudanças e difusões aceleradas, tecnologia da informação em ascensão, sendo que a concentração do conhecimento constitui a base da tecnologia e sua aplicação.

Para Porter (1999), as empresas conquistam uma posição de vantagem em relação aos melhores competidores do mundo em razão das pressões e dos desafios. Com a influência da globalização na disputa pelo mercado, as organizações buscam adotar medidas que surtam efeitos na capacitação de concorrência por preço, esforço de venda ou diferenciação de produtos para maximizar o desempenho. (FERRAZ; KUPFER; HAGUERNAUER, 1995).

O termo competitividade, entendido também como vantagem competitiva, pode ser compreendido como "a capacidade da empresa formular eimplementar estratégias concorrenciais que lhe permitam ampliar ou conservar, de forma duradoura, uma posição sustentável no mercado”. (FERRAZ; KUPFER; HAGUERNAUER, 1995 , p. 3).

Porter (1989; 1999) destaca que a vantagem competitiva é gerada por meio de um processo localizado, no qual aspectos sociais, como os valores nacionais, a cultura, estruturas econômicas, instituições e história contribuem para o êxito competitivo. Além desses aspectos sociais, a vantagem competitiva se consolida na maneira pela qual as organizações realizam suas atividades, conceituada pelo autor como cadeia de valores.

A cadeia de valor é composta pelas atividades primárias (operação) e de apoio (suporte). A ligação entre as atividades surge quando o desempenho de alguma atividade é afetado por outra,em que a integração entre essas atividades e a competência em administrá-las proporcionará uma vantagem competitiva à organização. (PORTER,1989; 1999).Esta cadeia de valores em um setor específico está inserida num fluxo mais amplo de atividades, que Porter (1989; 1999) denominou de sistema de valores. 
O sistema de valores inclui: fornecedores, para proporcionar insumos; empresa, atuante na transformação de insumos em produtos ou serviços; distribuição, por meio de canais de distribuição até o comprador; e, comprador, realizando suas próprias atividades com o produto comprado. (PORTER, 1989; 1999).

Figura 1: O sistema de valores

\section{Cadeia de valores do fornecedor}

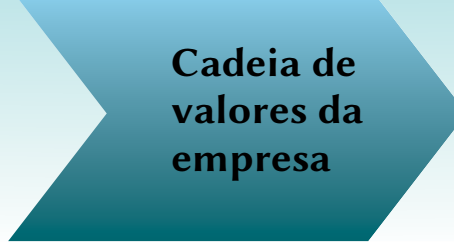

Cadeia de valores da empresa

Fonte: Porter (1989; 1999)

"A vantagem competitiva é, cada vez mais, função da competência com que uma empresa pode administrar todo esse sistema”. (PORTER, 1989,p.53).Portanto, a gestão global do sistema de valores assume um papel relevante para as organizações no âmbito competitivo, sendo percebida pela redução de custos, produção de bens e serviços com mais qualidade, velocidade e flexibilidade nas negociações e nas entregas. (VENTURA; MELO, 2013).

\subsection{Supply Chain}

\section{Management}

O Supply Chain Management (SCM) está passando por uma grande transformação. Anteriormente, estava focado na integração interna e agora está focada em forma de rede, desde seu primeiro fornecedor ao seu último cliente, com o intuito de alcançar níveis excelentes de desempenho.

O conceito SCM, considerado moderno na nova economia, incorpora diferenciação estratégica, melhoria de valor,melhoria da eficiência, redução de custos, integração da cadeia de suprimentos, excelência operacional e cadeias de suprimentovirtuais. (LUMMUS; VOKURKA,
1999; MENTZER et al., 2001; LAGROSEN, 2005; CHOW et al., 2008; MONCZKA et al., 2009; MELNYK et al., 2009; BIDGOLI, 2010; STOCK; BOYER, 2009).

O SCM é uma abordagem holística voltada à demanda, sourcing e procurement, produção e gestão de processos, como tambémé a formação de elos em uma rede constituída por suas partes, direta ou indiretamente envolvidas, que alcançam fabricantes, fornecedores, distribuidores, clientes e assim por diante, por meio da distribuição física, do fluxo de informações, financeiro, entre outros. (LUMMUS; VOKURKA, 1999; MENTZER et al., 2001; LAGROSEN, 2005; CHOW et al., 2008; MONCZKA et al., 2009; MELNYK et al., 2009; STOCK; BOYER, 2009; BIDGOLI, 2010; CORRÊA, 2010).

A gestão da cadeia de suprimentos é vista como um instrumento capaz de construir vantagem competitiva para a cadeia como um todo. Para que esse processo conduza o aumento da competitividade, é necessário que haja integração, cooperação e compartilhamento de informações, tanto dentro da organização como entre as organizações. (HUTT; SPEH, 2001). 
O princípio de uma abordagem de cadeia de suprimento refere-se,entre todos os integrantesque dela fazem parte, desde o primeiro fornecedor até o último cliente ou consumidor. Historicamente, os executivos concentravam-se em redução de custos de aquisição e de custos de fabricação, não importando-se com as despesas incorridas ao longo da cadeia logística. Isso acontecia devido à execução dos serviços logísticos serem desenvolvidos internamente, principalmente em depósitos, transportes e sistemas de informações. (BOWERSOX; CLOSS, 2010).

Essa maneira independente ao longo da cadeia de abastecimento conflitava com as expectativas dos clientes, como também com as metas de marketing, produção e distribuição. (BERTAGLIA, 2009). Nesse sentido, hoje existe a necessidade de que haja uma estrutura de cadeia de suprimentos que se alinhe com as características do produto e exigências do mercado.(FISHER,1997; VANDER VAART; VAN DONK, 2006).

Entretanto, Bowersox e Closs (2010) fomentam que houve uma reversão no sentido de desenvolvimento de cooperação na cadeia de suprimento. Os executivos perceberam que o ambiente propiciava conexões e relações com outras organizações, a fim de ambos obterem vantagens. (SUKATI, 2012). A disposição de alianças entrou em foco, com fornecedores globalizados ou não, que pudessem oferecer e contribuir na especialização e serviços logísticos de qualidade, a custos razoáveis. (BOWERSOX; CLOSS, 2010).

Bertaglia (2009) indica que,consequentemente, isso pode resultar em menores estoques, como, por exemplo, o varejo que busca baixar os níveis de estoque, demandando menores pedidos em maior frequência. Esse início de mudança penaliza o distribuidor, o atacadista e/ou o fabricante, que se obrigam a administrar seus custos de transporte e manter um fluxo adequado de informações para não obterem prejuízos nos processos, forçando-os a reformular um novo escopo estratégico. (SUKATI, 2012). Esforço estratégico este que deve integrar e coordenar toda a cadeia produtiva, a fim de gerar o melhor desempenho a todos os membros da própria cadeia. (WISNER, 2003; COHEN; ROUSSEL,2005; GREENJR.;WHITTEN; INMAN, 2008).

O fluxo de informação, outra ferramenta estratégica,está ligado ao movimento físico de produtos, materiais e também a todos os processos, como análises de pedidos, estimativas de vendas, planejamento de produção, compras e aquisições, capacidades, armazenagem e manuseio. Por isso, é de fundamental importância a presença de Tecnologia da Informação, pois ela proporciona maior confiabilidade ao processo. Assim, quanto maior e correto for o fluxo de informação na organização há mais facilidade de identificar as informações que realmente agregam valor aos processos, aumentando a velocidade e a confiabilidade das transações. Essas práticas de negócios são orientadas à redução de estoques na cadeia de abastecimento, buscando equilíbrio com o melhor atendimento às exigências de clientes e consumidores. (BERTAGLIA, 2009).

Sabe-se que a gestão da rede de suprimento é carente de estudos em decorrência da sua juventude na atuação das empresas. No entanto, essa área ganhou grande visibilidade e interesse na última década. A principal vertente desse interesse volta-se à tecnologia que, devido a sua rápida evolução, fez com que as empresas buscassem confiança, facilidade e integração com terceiros, delegando partes substanciais de seus processos produtivos e desenvolvimentos tecnológicos, a fim de alcançar resultados excepcionais em todos os elos. (CHOW et al., 2008; CORRÊA, 2010). 


\begin{tabular}{|c|c|c|}
\hline $\begin{array}{l}\text { Prioridades } \\
\text { competitivas }\end{array}$ & Conceito principal & Autores \\
\hline Custo & $\begin{array}{l}\text { Menor custo de produção e no } \\
\text { serviço prestado. }\end{array}$ & $\begin{array}{l}\text { Porter, 1985; Hayes e Pisano, 1996; Hutt e Speh, 2001; } \\
\text { Paiva et al., 2004; Moori, Ghobril e Marcondes, } 2009 .\end{array}$ \\
\hline Integração & $\begin{array}{l}\text { Em toda a rede de suprimento } \\
\text { desde o primeiro fornecedor até } \\
\text { o último cliente. }\end{array}$ & $\begin{array}{l}\text { Porter, 1985; 1989; Stevens, 1990; Turner, 1993; Stock, } \\
\text { Greis e Kasarda, 1998; Narasimhan e Jayaram, 1998; } \\
\text { Stock, Greis e Kasarda, 2000; Corrêa, } 2010 .\end{array}$ \\
\hline Flexibilidade & $\begin{array}{l}\text { Em mix, volume, troca e varie- } \\
\text { dade de produtos e serviços no } \\
\text { sistema produtivo. }\end{array}$ & $\begin{array}{l}\text { Porter, 1985; Gerwin, 1987; Cox Jr., 1989; Hayes e } \\
\text { Pisano, 1996; Hutt e Speh, 2001; Paiva et al., 2004; } \\
\text { Moori, Ghobril e Marcondes, } 2009 .\end{array}$ \\
\hline Velocidade & $\begin{array}{l}\text { Nos processos e na entrega } \\
\text { dando uma resposta rápida e } \\
\text { eficiente ao consumidor. }\end{array}$ & Paiva et al., 2004; Moori, Ghobrile e Marcondes, 2009. \\
\hline Qualidade & No produto e serviço. & $\begin{array}{l}\text { Porter, 1985; De Meyer et al., 1989; Hayes e Pisano, } \\
\text { 1996; Paiva et al., 2004; Moori, Ghobrile Marcondes, } \\
\text { 2009. }\end{array}$ \\
\hline Preço & $\begin{array}{l}\text { Melhor preço em relação ao bem } \\
\text { oferecido. }\end{array}$ & Omta et al., 2001; Paiva et al., 2004. \\
\hline Inovação & $\begin{array}{l}\text { No produto, serviço ou pro- } \\
\text { cesso, proporcionando maior } \\
\text { competitividade. }\end{array}$ & Porter, 1989; Paiva et al., 2004. \\
\hline
\end{tabular}

Fonte: Dos autores (2014)

a) Custo: vinculado as mais diversas oportunidades de compra que o globo oferece, pode-se destacar que quanto menor o custo em relação à redução de estoques na empresa, manutenção da produção em ritmo constante, monitoramento sistemático dos custos de produção e

o controle de produtividade dos operários maior são fatores que elevam o nível de competitividade da organização. (PORTER, 1985; HAYES; PISANO, 1996; HUTT; SPEH, 2001; PAIVA et al., 2004; MOORI; GHOBRIL; MARCONDES, 2009).

b) Integração: a integração da cadeia de suprimentos pode ser conceituada pelo grau de integração apresentada por todas as atividades dentro de uma organização entre fornecedores e clientes, formando, assim, uma rede integrada de suprimentos, bem como apresentar uma comunicação eficaz entre todos os membros pertencentes à cadeia. Nesse sentido, para obter vantagem competitiva perante outras redes, é necessário existir integração entre todos os "nós" envolvidos na rede, com ligações fortes entre seus elos. (PORTER, 1989; STEVENS, 1990; TURNER, 1993; STOCK; GREIS; KASARDA, 1998; NARASIMHAN; JAYARAM, 1998; STOCK; GREIS; KASARDA, 2000; CORREAA, 2010). 
c) Flexibilidade: para que uma rede de suprimentos seja flexível, destacam-se vários elementos a fim de obter vantagem competitiva: sistema que permite flexibilidade e troca da sequência de produção; mudanças rápidas em projetos e no mix de produtos; fácil e rápido ajuste do volume de produção quando há mudança no volume de pedidos; variedade no oferecimento de linhas de produtos (várias famílias de produtos); vários produtos diferentes em cada linha de produto que possui (diversidade em cada linha), o que permite introdução rápida de novas versões de produtos existentes ou de produtos totalmente novos. (PORTER, 1985; GERWIN, 1987; COX JR., 1989; HAYES; PISANO, 1996; HUTT; SPEH, 2001; PAIVA et al., 2004; MOORI; GHOBRIL; MARCONDES, 2009).

d) Velocidade: atender aos pedidos nas datas e nas quantidades da entrega planejada; entregar os produtos mais rapidamente que os principais concorrentes; e entregar sempre dentro do prazo. $\mathrm{Ou}$ seja, refere-se à diminuição no tempo de entrega de um determinado produto ou serviço solicitado pelo cliente externo. Para alcançar este fator competitivo, é preciso diminuir o tempo no processo da manufatura, o que irá beneficiar a empresa e sua operação a ponto de deixá-la mais enxuta e produtiva, alcançando a vantagem competitiva com mais facilidade. (PAIVA et al., 2004; MOORI; GHOBRIL; MARCONDES, 2009). e) Qualidade: são levados em consideração aspectos condizentes com as obrigações das empresas, a fim de galgar maior vantagem competitiva.Destacam-seemoferecerprodutos com características e funcionalidades superiores ou não disponibilizadas pelos concorrentes; oferecer produtos fabricados de acordo com padrões pré-estabelecidos; oferecer produtos com baixa taxa de defeitos eque sejam duráveis; oferecer produtos com qualidade uniforme (tamanho, sabor, aparência, textura); oferecer produtos que cumpram as especificações técnicas (pureza e composição); e oferecer serviços complementares aos clientes (pós-venda, serviço de atendimento ao consumidor SAC, reposição etc.). (PORTER, 1985; DE MEYER et al., 1989; HAYES; PISANO, 1996; PAIVA et al., 2004; MOORI;GHOBRIL;MARCONDES, 2009).

f) Preço: as negociações globais não se delimitam apenas em questões relativas a preço, mas a um conjunto de atividades eficientes de prioridades que venham satisfazer por completo as necessidades dos clientes. Tal conjunto possibilita galgar maiores vantagens sobre outras organizações ou redes, pois a capacidade de oferecer bens tangíveis e/ou intangíveis com preços mais baixos comparados aos da concorrência possibilitam maior competitividade no mercado. (OMTA et al., 2001; PAIVA et al., 2004).

g) Inovação: a inovação tornou-se cada vez mais relevante para as organizações. Empresas buscam inovações por meio de novos ou modificados produtos, serviços e processos, a fim de diferenciarse de seus concorrentes de baixo custo e de alta qualidade. (PORTER, 1989; PAIVA et al., 2004). 
Diante desse levantamento teórico, propõe-se o modelo operacional considerando os efeitos da globalização e avanços tecnológicos sobre o sistema de valor - SCM. Todavia, defronte a rivalidade acirrada entre organizações, o modelo propõe que o SCM adote alguma prioridade competitiva perante as lacunas oportunas existentes, para obter vantagens sobre os concorrentes. Por sua vez, de forma cíclica, as prioridades competitivas influenciam a globalização e os avanços tecnológicos, nos quais proporcionam um ambiente organizacional dinâmico e mutável.

Figura 2: Modelo operacional

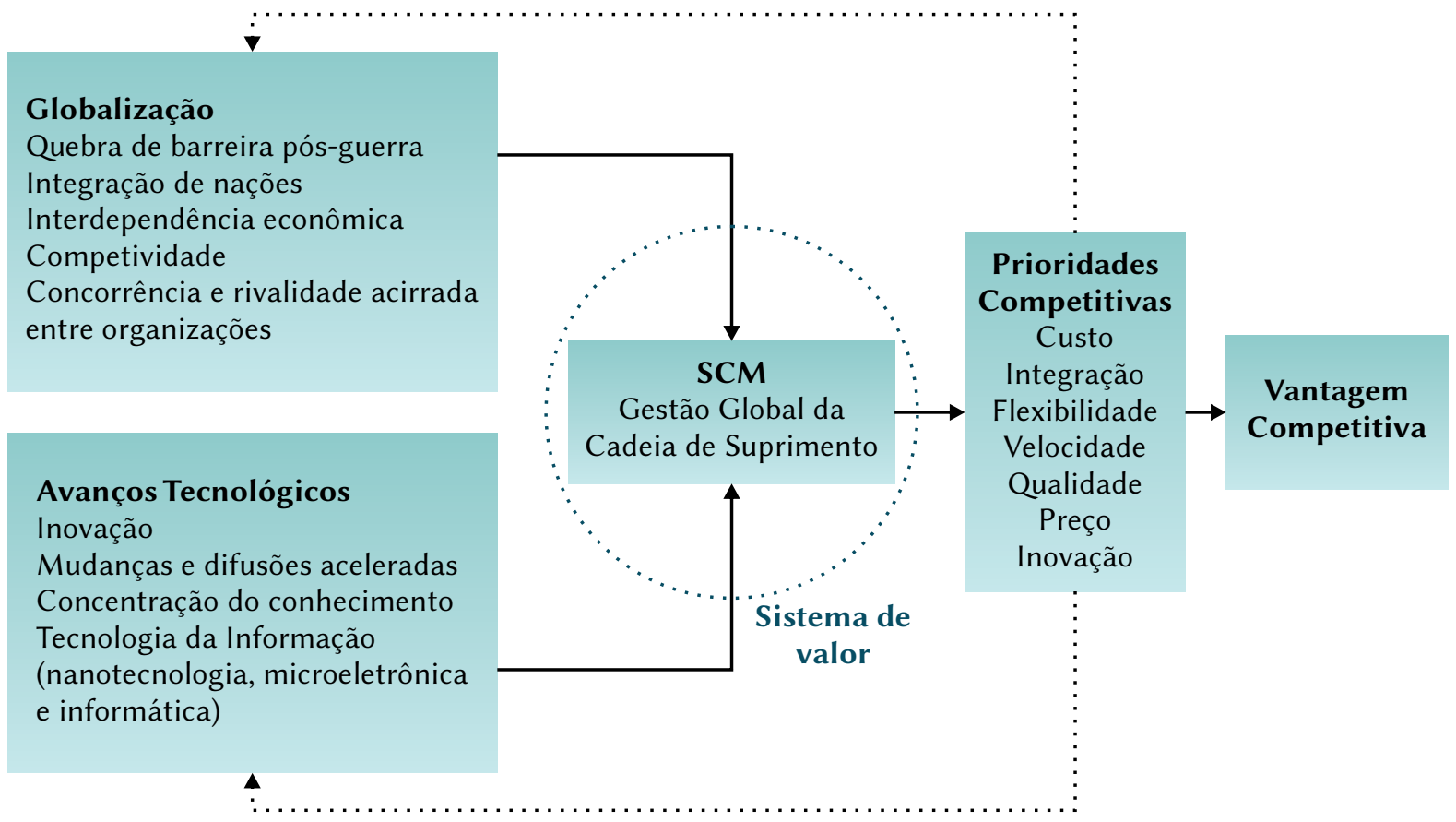

Fonte: Dos autores (2014)

A vantagem competitiva surge, fundamentalmente, a partir das condições que a gestão global da cadeia de suprimento possui em obter constante capacidade de ampliar ou manter uma posição sustentada no mercado, conforme concorrência. (PORTER, 1989; FERRAZ; KUPFER; HAGUERNAUER, 1995; MOTTA, 1995; PORTER, 1999).
Diante do modelo apresentado, tem-se, a partir deste estudo, a proposta de testar a seguinte hipótese:

H1 - A gestão global da cadeia de suprimento influencia as prioridades competitivas na busca por vantagem competitiva. 


\section{DELINEAMENTO METODOLÓGICO}

A pesquisa realizou-se por meio de abordagem quantitativa, a qual se caracteriza pelo emprego de instrumentos estatísticos nas modalidades de coleta e tratamento dos dados, como o percentual, desvio-padrão, média, ou por meio de cálculos mais complexos, como coeficiente de correlação, análise de regressão, entre outros. (RICHARDSON,1999; RAUPP;BEUREN, 2004; HAIR JR. et al., 2005).

Utiliza-se como delineamento metodológico a pesquisa explicativa, que se constitui mais complexa, pois registra, analisa, classifica e interpreta os fenômenos estudados, e procura identificar seus fatores determinantes. A pesquisa explicativa procura a razão e o porquê das coisas, pois integra estudos mais aprofundados pela necessidade de explicar os determinantes na ocorrência dos fenômenos. (ANDRADE, 2002; RAUPP; BEUREN, 2004).

O procedimento de pesquisa utilizado no estudo é o multicaso, pois se refere a um conjunto de estudos de caso. Triviños (1987) afirma que os estudos multicasos possibilitam estudar dois ou mais sujeitos. Yin (1994) argumenta que é uma pesquisa de natureza empírica, em que investiga-se o fenômeno dentro da situação na qual ele ocorre, sendo adequado em pesquisas quando os fenômenos e os contextos estudados não são claros.

A coleta de dados é realizada por meio de questionário constituído por um conjunto ordenado de perguntas que devem ser respondidas pelo pesquisado. Portanto, é um instrumento cientificamente desenvolvido para medir características importantes de indivíduos, empresas, eventos e outros fenômenos. (COLAUTO; BEUREN, 2004; HAIR JR. et al., 2005).
O questionário foi desenvolvido de forma estruturada, com questões fechadas, pois permite ao respondente escolher a resposta que melhor evidencia a sua situação ou seu ponto de vista. (COLAUTO; BEUREN, 2004). Para ter acesso aos dados, foi realizado contato telefônico com os respondentes. Em seguida, foram disponibilizadas as questões em formulários desenvolvidos via internet com auxílio da ferramenta Google Docs, o que propiciou uma resposta sem contato pessoal.

Os dados foram coletados e mensurados por meio da escala Likert. Essa escala varia entre cinco categorias de respostas, que vão de "discordo totalmente" até "concordo totalmente", exigindo que os participantes indiquem o grau de concordância ou discordância de acordo com cada questionamento. (MALHOTRA, 2001).

A população da pesquisa corresponde a três empresas multinacionais do segmento metalmecânico, na manufatura de transformadores (geradores de energia), situados no Estado de Santa Catarina, Brasil. Para Colauto e Beuren (2004), a população consiste na totalidade de elementos distintos com características semelhantes, definidos para determinado estudo.

A amostra é representada por todos os profissionais da área de Logística Internacional e Comércio Exterior das empresas investigadas,o que abrangeu trinta respondentes, dos quais seis foram excluídos devido às inconformidades das respostas. Ou seja, representa um subconjunto da população, selecionada em conformidade com as características do objeto de pesquisa. (COLAUTO; BEUREN, 2004; HAIR JR. et al., 2005). 
No estudo foi identificada uma hipótese de pesquisa: H1 - A gestão global da cadeia de suprimento influencia as prioridades competitivas na busca por vantagem competitiva. De acordo com Hair Jr. et al. (2005), as hipóteses possibilitam que os pesquisadores expliquem e testem ideias preliminares em relação aos fenômenos propostos. Ainda, possuem como função a orientação dos pesquisadores na condução do estudo. (PORTON; BEUREN, 2004).

A análise de dados foi realizada a partir do material obtido durante o processo investigatório, por meio de questionário, o qual é organizado a fim de identificar as principais tendências e os padrões que se apresentam como relevantes. (COLAUTO; BEUREN,2004). Como ferramenta de auxílio na análise de dados, utilizou-se o software SPSS for Windows - versão 21, que, segundo Pereira (2004) e Wagner, Motta e Dornelles (2004), é uma ferramenta de informática que permite realizar análises estatísticas complexas, manipular e visualizar dados, egerar tabelas e gráficos que resumem esses dados. Os testes utilizados no estudo foram estatística descritiva de frequência e teste não paramétrico qui-quadrado $\left(\mathrm{X}^{2}\right)$.

\section{ANÁLISE DOS DADOS E RESULTADOS}

Quanto às características do perfil da amostra, identificou-se uma leve tendência a respondentes do gênero feminino, com $54,20 \%$, enquanto 45,80\% são do gênero masculino. Em termos de idade, a maior concentração dos respondentes possui entre dezoito e 31 anos, com uma representatividade de 62,50\%. Referente aos cargos dos respondentes, foram envolvidos todos os níveis hierárquicos de logística e áreas relacionadas das empresas pesquisadas, desde diretor até assistente administrativo logístico.

Constatou-se, ainda, que 50\% dos profissionais pesquisados trabalham menos de dois anos na empresa, e que a grande maioria desses profissionais (54,10\%) desempenham suas atividades também a menos de dois anos na área logística e comércio exterior. Vale salientar que, entre os entrevistados, 79,20\% são da área de logística e 20,80\% da área de comércio exterior.

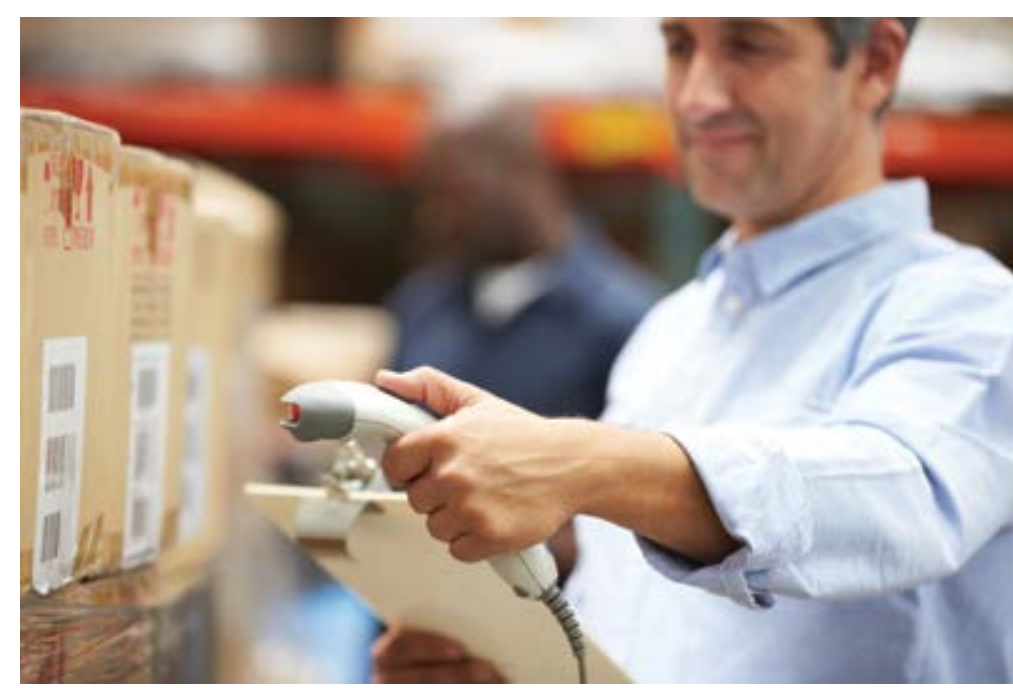

A globalização apresentou-se como um expoente de interdependência econômica entre as nações, desde o fluxo de bens, serviços, capital, até o conhecimento e informações. (JANSEN; ROTONDARO;JANSEN,2005; HOSKISSON et al., 2009). Isso fez com que a concorrência e a incerteza do mercado 
surgissem como pressupostos ao mercado mundial; ou seja, as empresas que se adaptassem a esse novo cenário, alcançando um melhor desempenho, estariam condicionadas a obter maior vantagem competitiva. (HOSKISSON et al., 2009; PORTER, 1989; 1999).

Para tal assertiva da pesquisa, aplicou-se o teste qui-quadrado e constatou-se que a globalização contribui para a formação da cadeia global de suprimentos (CadGlob), apresentando $p$-value 0,000, bem como os investimentos em avanços tecnológicos também condicionam uma maior vantagem competitiva (AvTecn), com $p$-value 0,000 . Tal teste é alicerçado por Hair Jr. et al. (2005), no que tange a significância estatística entre as distribuições de frequência de dois ou mais grupos, de acordo com a tabela 1 .

Tabela 1: Globalização e vantagem competitiva

\begin{tabular}{l|c|c|c|c|c|c}
\hline & Concorr & PosSust & CadGlob & AvTecn & InvTecn & SistVal \\
\hline Qui-quadrado & 1,500 & 5,250 & 38,000 & 23,250 & 6,833 & 2,250 \\
\hline P-value & 0,221 & 0,072 & 0,000 & 0,000 & 0,145 & 0,325 \\
\hline
\end{tabular}

Fonte: Dos autores (2014)

Em relação às variáveis concorrência acirrada no mercado global (Concorr); posição sustentada no mercado pela empresa (PosSust); existência de investimento tecnológico na empresa e não em toda cadeia (InvTecn); e ampliação da cadeia para um sistema de valor (SistVal), não há evidências suficientes para afirmar que esses fatores não influenciam a gestão global da cadeia de suprimento, devido os $p$-values possuírem significância acima de 0,05.

Em resposta à hipótese 1 (H1), adotou-se como método o teste Qui-quadrado e o teste descritivo, que para Hair Jr. et al. (2005), são usados para avaliar as diferenças estatísticas entre duas distribuições.

Tabela 2: Adoção de prioridades competitivas

\begin{tabular}{|l|c|c|c|c|c|c|c}
\hline \multicolumn{1}{|c|}{ Adoção a: } & Custo & Integração & Flexibilidade & Velocidade & Qualidade & Preço & Inovação \\
\hline $\mathbf{X}^{2}$ & 15,00 & 15,67 & 9,00 & 27,33 & 40,17 & 35,17 & 7,00 \\
\hline Significância & 0,000 & 0,000 & 0,029 & 0,000 & 0,000 & 0,000 & 0,072 \\
\hline
\end{tabular}

Fonte: Dos autores (2014)

Diante dos dados da pesquisa, há evidências de que a gestão global da cadeia de suprimentos adota prioridades na busca por vantagem competitiva, conforme as variáveis Custo, com $p$-value 0,002 ; Integração, com $p$-value 0,001; Flexibilidade, com $p$-value 0,029; e
Velocidade, Qualidade e Preço, com $p$-values 0,000 . Portanto, são prioridades adotadas pela gestão global da cadeia de suprimentos na busca por vantagem competitiva, na percepção dos colaboradores entrevistados nas empresas. 
Quanto à variável Inovação, $\operatorname{com} p$-value 0,072 , não há evidências suficientes para afirmar que a gestão global da cadeia de suprimentos adota prioridade na busca por vantagem competitiva, considerando um nível de confiança de 95\% de significância.Essa descoberta demonstra-se inconsistente com estudos anteriores.(PORTER, 1989; PAIVA et al., 2004).

Tabela 3: Descrição das médias das prioridades competitivas

\begin{tabular}{l|c|c|c|c|c}
\hline \multicolumn{7}{c}{ Estatística descritiva } \\
\hline Adoção & N & Média & Desvio Padrão & Mínimo & Máximo \\
\hline Custo & 24 & 4,21 & 1,215 & 1 & 5 \\
\hline Integração & 24 & 3,96 & 0,751 & 2 & 5 \\
\hline Flexibilidade & 24 & 4,17 & 0,868 & 2 & 5 \\
\hline Velocidade & 24 & 4,50 & 0,885 & 1 & 5 \\
\hline Qualidade & 24 & 4,46 & 1,062 & 1 & 5 \\
\hline Preço & 24 & 4,42 & 1,06 & 2 & 5 \\
\hline Inovação & 24 & 4,08 & 1,018 & 2 & 5 \\
\hline
\end{tabular}

Fonte: Dos autores (2014)

Para apresentar e enlaçar com mais precisão a análise da pesquisa, verificou-se, com um teste estatístico descritivo das prioridades estudadas, e constatou-se que as maiores médias de adoção das prioridades são verticalizadas pelos menores valores de significância (o que traz confiabilidade), conforme visualizadas nas tabelas 2 e 3 .

Portanto,entende-se que os representantes empresariais dos setores pesquisados adotam as sete prioridades competitivas para a gestão global da cadeia de suprimentos, a fim de buscarvantagem competitiva. No entanto, enfaticamente, disponibilizam maiores importâncias para os aspectos prioritários de Velocidade, Qualidade e Preço. Essas evidências corroboram com as prioridades competitivas sugeridas, como custo, integração, preço, velocidade, qualidade, flexibilidade e inovação, conforme mencionado pelos autores anteriormente citados que fundamentam esta pesquisa, são atributos fundamentais para a elevação da competitividade da organização.

De acordo com Bowersox e Closs (2010), historicamente, os executivos não se preocupavam com a integração de toda cadeia de valor. No entanto, ao longo da história, essa concepção mudou, e, conforme Porter (1985), considera-se que a integração proporciona vantagem competitiva. Atualmente, a gestão cadeia de suprimentos preocupa-se com todos os integrantes, desde o primeiro fornecedor até o último cliente ou consumidor.(BOWERSOX; CLOSS, 2010; SUKATI, 2012).

No que tange a gestão global da cadeia de suprimentos das empresas pesquisadas, notou-se alto grau de importância acerca da capacidade de construir vantagem competitiva para todo o sistema por meio da integração, cooperação e 
compartilhamento de informações, tanto dentro da organização como entre as organizações, como mencionam Hutt e Speh (2001).

Perante os resultados obtidos que podem ser visualizados na tabela 3 , a gestão da cadeia de suprimentos tem apresentado, como indicam Bowersox e Closs (2010), a presença de conexões e relações com outras organizações, a fim de obterem vantagens por meio de cooperação, integração e compartilhamento de informações, que são fundamentais para a sobrevivência das empresas e impulsionados por esta cadeia. (BOWERSOX; CLOSS, 2010; SUKATI, 2012; WISNER, 2003; COHEN; ROUSSEL,2005; GREENJR.;WHITTEN; INMAN, 2008).

Tabela 4: Média das variáveis

\begin{tabular}{l|c|c|c|c|c}
\hline Descrição & N & Mínimo & Máximo & Média & DesvioPadrão \\
\hline CompartInf & 24 & 3 & 5 & 4,04 & 0,464 \\
\hline CoopInf & 24 & 3 & 5 & 4,08 & 0,584 \\
\hline IntegrInf & 24 & 3 & 5 & 4,17 & 0,637 \\
\hline DesempEmpr & 24 & 2 & 5 & 4,21 & 0,779 \\
\hline
\end{tabular}

Fonte: Dos autores (2014)

Da mesma forma, a cooperação, o compartilhamento e a integração das informações entre os atores da cadeia de suprimentos conduzem o aumento da competitividade. (HUTT; SPEH, 2001). Assim, evidenciou-se na pesquisa, pelas médias dos respondentes, que o compartilhamento de informações (CompartInf), com média de 4,04; a cooperação de informações (CoopInf), com média de 4,08; e a integração de informações (IntegrInf), com média de 4,17, são predicados funcionais para o desempenho (DesempEmpr) da gestão global da cadeia de suprimentos nas empresas pesquisadas.

Portanto, constatou-se que as três variáveis em estudo possuem determinado grau de importância para o aumento do desempenho da gestão global da cadeia de suprimentos, conforme percepção dos profissionais das empresas pesquisadas. 


\section{CONSIDERAÇÕES FINAIS}

Pormeiodaglobalização,observa-setransformações intensas no campo organizacional paraumanova dinâmicaeconômica.(MOTTA,1995;PESSANHA et al., 2012). A influência da alta concorrência fez as empresas entenderem as mudanças como algo rotineiro e necessário, evoluindo de um enfoque interno para a integração de um sistema de valor. (HOSKISSON et al.,2009).

Constatou-se na presente pesquisa que a globalização contribui para a formação da cadeia global de suprimento, bem como para os investimentos em avanços tecnológicos, os quais trazem maior vantagem competitiva para as organizações, como também afirmam Hoskisson etal. (2009) e Porter (1985).

Quanto à adoção das prioridades competitivas pela gestão global da cadeia de suprimentos (H1), verificou-se que o custo, a integração, a flexibilidade, a velocidade, a qualidade e o preço podem ser atributos que a cadeia adota a fim de obter vantagem competitiva. Conforme Correa (2010) e Paiva et al. (2004), esses atributos condicionam a organização a alcançar competitividade.

No entanto, o atributo inovação, rejeitado pelo teste qui-quadrado,nãoé entendido como aspecto obrigatório pelas organizações, por se diferenciarem dos atributos supracitados. Ou seja, custo, integração, flexibilidade, velocidade, qualidade e preço são entendidos pelas empresas pesquisadas como essenciais e indiscutíveis para a busca de vantagem competitiva, assim como aponta Porter (1985; 1989). Essa evidência não repreende a inovação, apenas a demonstra de forma contrária a resultados anteriores, de acordo com estudos de Porter(1989)e Paiva etal.(2004), sendonecessário novos estudos.

Além disso, constatou-se, por um teste estatístico e descritivo, que das sete prioridades validadas e estudadas em questão, todas são adotadas e dadas comoimportantespelosrepresentantesempresariais dossetorespesquisados. No entanto,enfaticamente, disponibilizam-se maiores importâncias e adoção paraosaspectos prioritários develocidade,qualidade e preço, a fim de buscar vantagem competitiva, tal como evidencia Porter $(1985 ; 1989)$.

Quanto à existência de influências das prioridades competitivasnodesempenhodagestãoglobaldacadeia de suprimentos, a fim de responder à pergunta de pesquisa, identificou-seque podehaver influências das prioridades de custo, integração, flexibilidade, preço, velocidade e qualidade. Pois, de acordo com Ferraz, Kupfer e Haguernauer (1995), por efeito da globalização, a todo instante as organizações buscam adotar medidas com vistas a maximizar seu desempenho. A prioridade inovação, estatisticamente, ficou abaixo dos requisitos de confiança (95\%) para afirmar como uma prioridade adotada pelas empresas. No entanto, o teste descritivo representado na tabela 3 destaca a inovação como relevante para obtenção de vantagem competitiva. Tal fato ocorre pela variação de percepção dos respondentes.

Ainda em resposta à pergunta de pesquisa, velocidade, qualidade e preço foram diagnosticados como as prioridades mais importantes e influenciadoras nodesempenho da gestão globalda cadeia de suprimento das organizações multinacionais em estudo. Nessas condições, Ventura e Melo (2013) elucidam que a gestão global do sistema de valor assume um papel relevante para as organizações no fator competitivo, sendo formada sob a influência de prioridades competitivas.

Deacordocomosdadosdapesquisa evidenciadosna tabela4,observa-sequeaintegraçãodasinformações entre os membros do sistema de valor condiciona melhor desempenho para toda a cadeia, com uma média de 4,17 entre os respondentes, assim como enfatizado pelos autores Wisner (2003), 
Cohen e Roussel (2005), Green Jr., Whitten e Inman (2008) e Sukati (2012). Contudo, a cooperação e o compartilhamento das informações podem influenciar o aumento do desempenho da gestão global da cadeia de suprimentos, conforme dados da pesquisa. Embora a mudança necessite que todos os membros se engajem para que, de fato, obtenham melhores desempenhos. (HUTT; SPEH, 2001).
A pesquisa limita-se pelo envolvimento de apenas uma área/setor na empresa (logística/ comércio exterior), bem como sua amostra, sendo em organizações multinacionais. Como sugestão para novas pesquisas, recomenda-se a extensão a outras áreas, assim como outras configurações de organizações, além da busca por compreender como ocorre a adoção de prioridades competitivas para melhor desempenho da gestão global da cadeia de suprimento.

\section{REDE SENAI DE INOVAÇÃO E TECNOLOGIA}

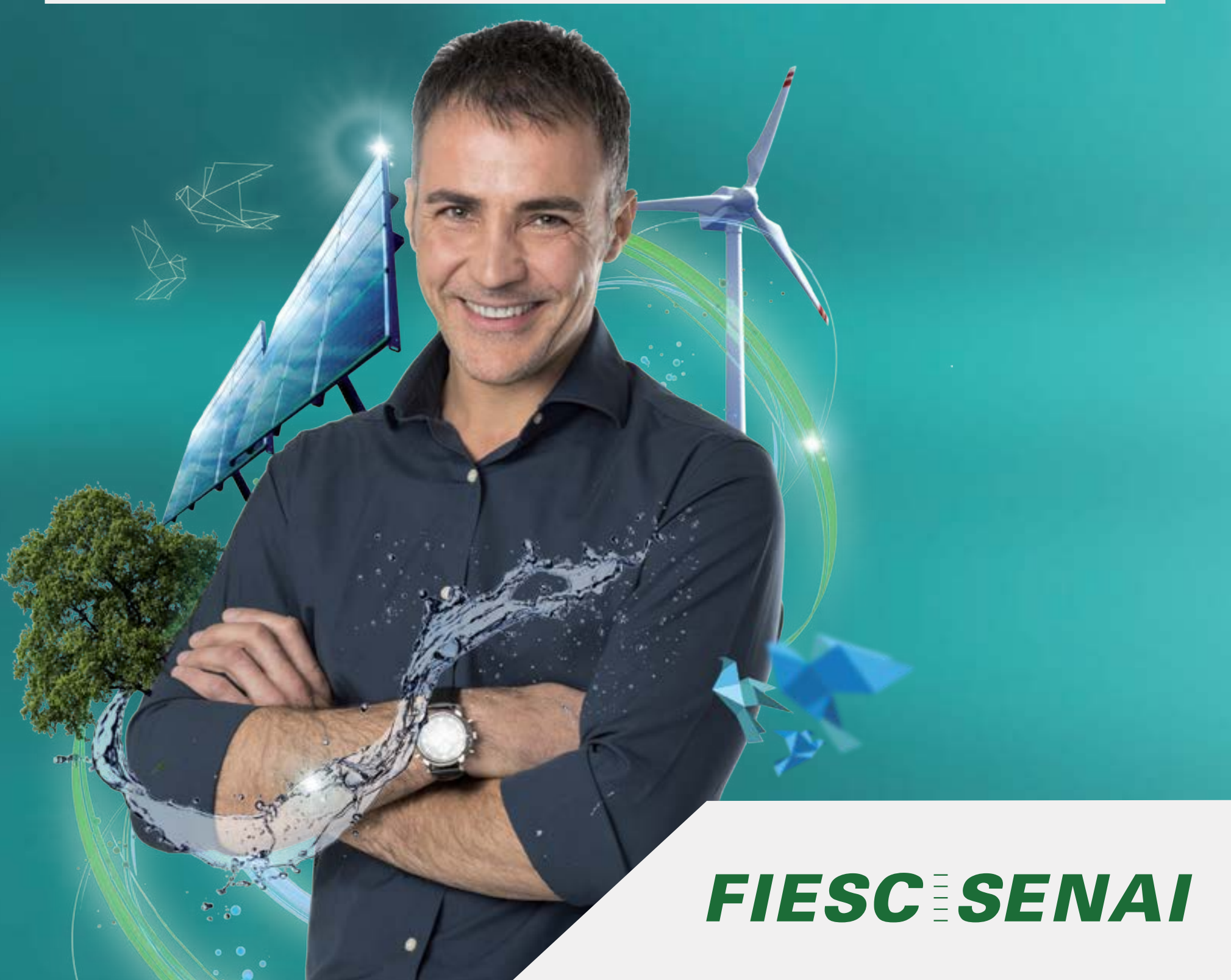




\section{THE INFLUENCE}

OF COMPETITIVE

PRIORITIES

IN GLOBAL

SUPPLY CHAIN

MANAGEMENT - A

MULTI-CASE STUDY

\begin{abstract}
Globalization has influenced the competitive growth, breaking down barriers and providing greaterchallenges in organizations. The adoption of new competitive priorities was necessary to get competitive advantage. The objective of this research is to identify the degree of importance and the impact of competitive priorities for the overall management of the supply chain in pursuit of competitive advantage which is demonstrated in the bypothesis of this research. The methodologicalapproach is called quantitative explanatory, with multi-case study at three multi-national organizations in the metal-mechanic sector, located in the State of Santa Catarina. As a result we identified the confirmation of the hypothesis; priorities of cost, integration, speed, flexibility, quality and price can influence the performance of global supply chain management; and integration, cooperation and sharing of information in the supply chain affect a better performance in the value system.
\end{abstract}

\section{REFERÊNCIAS}

ANDRADE, M. M. de. Como preparar trabalhos para cursos de pós-graduação: noções práticas. 5 . ed. São Paulo: Atlas, 2002.

BALANCO, P. A dialética da globalização e a supressão do pré-capitalismo. Revista de Economia. v. 34, n. 4, p. 183-203, 2008.

BERTAGLIA, P. R. Logística e gerenciamento da cadeia de abastecimento. São Paulo: Saraiva, 2009.

BIDGOLI, H. The handbook of technology management: Supply chain management, marketing and advertising, and global management. 2. ed. New Jersey: John Wiley and Sons, 2010.

BOWERSOX, D. J.; CLOSS, D. J. Logística empresarial: o processo de integração da cadeia de suprimento. São Paulo: Atlas, 2010.

CHOW, W. S. et al. Supply chain management in the US and Taiwan: An empirical study. The International Journal of Management Science. v. 36, p. 665-679, 2008.

COHEN, S.; ROUSSEL, J. Strategic Supply Chain Management: The Five Disciplines for Top Performance. Boston: McGraw-Hill, 2005.

COLAUTO, R. D.; BEUREN, I. M. Coleta, Análise e Interpretação dos dados. In: BEUREN, I. M. (org.). Como elaborar trabalhos monográficos em contabilidade: teoria e prática. 2. ed. São Paulo: Atlas, 2004.

CORRÊA, H. L. Gestão de Redes de

Suprimentos: Integrando Cadeias de Suprimentos. 3 ed. São Paulo: Atlas, 2010.

COX JR., T.J. Toward the Measurement of Manufacturing Flexibility.

Production and Inventory Management Journal, v. 30, n. 1, p. 68-72, 1989.

DE MEYER, A. et al. Flexibility: The Next Competitive Battle. The Manufacturing Futures Survey. Strategic Management Journal, v. 10, p. 135-144, 1989.

ERTURGUT, R. The Future of Supply Chain and Logistics Management in the Strategic Organizations: Contractor Companies and New Generation Suppliers. Procedia-Social and Behavioral Sciences, v. 46, p. 4221-4225, 2012.

FERRAZ, J. C.; HAGUERNAUER, L; KUPFER, D. Made in Brazil: desafios competitivos para a indústria. Rio de Janeiro: Campus, 1995. 
FISHER, M. L. What is the right supply chain for your product? Harvard Business Review, v. 75, n. 2, p. 105-117, 1997.

GERWIN, D. An Agenda for Research on the Flexibility of Manufacturing Process. International Journal of Operations \& Production Management, v. 7, n. 1, p. 38-49, 1987.

GREEN JR., K. W.; WHITTEN, D.; INMAN, A. The impact of logistics performance on organizational performance in a supply chain context. Supply Chain Management, v. 13, n. 4, p. 317-327, 2008.

GUIDO, A. L. B.; LIMA, R. T. de. Empresas transnacionais e internacionalização: uma análise bibliométrica dos termos. Revista Brasileira de Administração Científica, v. 3, p. 83, 2012.

HAIR JR, J. F. et al. Fundamentos de métodos de pesquisa em administração. Porto Alegre: Bookman, 2005.

HAYES, R.; PISANO, G. Manufacturing strategy at the intersection of two paradigm shifts. Produtions and operations management, v. 5, n. 1, p. 25-41, 1996.

HOSKINSSON, R. E. et al. Estratégia competitiva. São Paulo: Cengage Learning, 2009.

HUTT, M. D.; SPEH, T. W. B2B Gestão de Marketing em Mercados Industriais e Organizacionais. 7 ed. Porto Alegre: Bookman, 2001.

HULT, T. M.; KETCHEN, D. J.; ARRFELT, M. Strategic supply chain management: improving performance through a culture of competitiveness and knowledge development. Strategic Management Journal, v. 28, n. 10, p. 1035-1052, Out, 2007.

HULT, T. M., KETCHEN, D. J., SLATER, S. F. Information processing, knowledge development, and strategic supply chain performance. Academy of Management Journal. v. 47, n. 2, p. 241-253, 2004.

JANSEN, L. K. C.; ROTONDARO, J. R. G.; JANSEN, J. U. Estratégias de sobrevivência para pequenas e médias empresas em ambientes globalizados: um estudo de caso do setor eletroeletrônico. Gestão e Produção. v. 12, n. 3, p. 405-416, 2005.

$\mathrm{KOH}, \mathrm{L}$. et al. The impact of supply chain management practices on performance of SMEs. Industrial Management \& Data Systems, v. 107, n. 1, p. 103-124, 2007.
LAGROSEN, S. Customer involvement in new product development: A relationship marketing perspective. European Journal of Innovation Management, v. 8, n. 4, p. 424-436, 2005.

LI, S. et al. Development and validation of a measurement instrument for studying supply chain management practices. Journal of Operations Management. v. 23, p. 618-641, 2005.

LUMMUS, R. R., VOKURKA, R. J. Defining supply chain management: A historical perspective and practical guide lines. Industrial Management \& Data Systems, v. 99, n. 1, p. 11-17, 1999.

MALHOTRA, N. K. Pesquisa de marketing: uma orientação aplicada. 3. ed. Porto Alegre: Bookman, 2001.

MELNYK, S. A. et al. Mapping the future of supply chain management: A Delphi study. International Journal of Production Research, v. 47, n. 16, p. 46294653, 2009.

MENTZER, J. T.; DE WITT, W.; KEEBLER, J. S. Defining supply chain management. Journal of Business Logistics, v. 22, n. 2, p. 1-25, 2001.

MONCZKA, R. M. et al. Purchasing and supply chain management. 4. ed. United States of America: Cengage Learning. 2009.

MOORI, R. G.; GHOBRIL, A. N.; MARCONDES, R. C. Alignment of competitive priorities between sellers and buyers of machines and equipments. RAM - Revista de Administração Mackenzie, v. 10, n. 4, p. 156-182, 2009.

MOTTA, R. A busca da competitividade nas empresas. Revista de Administração de empresas. São Paulo, v. 35, n. 1, p. 12-16, Mar./Abr., 1995.

NARASIMHAN, R.; JAYARAM, J. Causal linkages in supply chain management: an exploratory study of north american manufacturing firms. Decision Sciences, v. 29, n. 3, p. 579-605, 1998.

OBADIA, I. J.; VIDAL, M. C. R.; MELO, P. F. F. Uma abordagem adaptativa de intervenção para mudança organizacional. Gestão e Produção. v. 14, n. 1, p. 125-138, 2007.

OMTA, S.W. F. et al. Chain and Network Science: a research framework. Journal on Chain and Network Science, v.1, n. 1, p. 1-6, 2001.

PAIVA, E. L. et al. Estratégia de Produção e de Operações: Conceitos, Melhores Práticas e Visão de Futuro. Porto Alegre: Bookman, 2004. 
PEREIRA, A. Guia prático de utilização do SPSS: análise de dados para ciências sociais e psicologia. 5 . ed. Lisboa: Sílabo, 2004.

PESSANHA, G. R. G. et al. Impactos das estratégias de fusão e aquisição na rentabilidade dos bancos adquirentes: uma aplicação dos modelos de intervenção no setor bancário brasileiro. RAM, Revista de Administração Mackenzie. v. 13, n. 5, p. 101-134, 2012.

PORTER, M. E. Competitive advantage: creating and sustaining competitive performance. New York: Free Press, 1985.

A vantagem competitiva das nações. Rio de Janeiro: Campus, 1989.

Competição $=$ On competition: estratégias competitivas essenciais. 8. ed. Rio de Janeiro: Campus,1999.

PORTON, R. A. de B.; BEUREN, I. M. Formulação de hipóteses e caracterização de variáveis em pesquisa contábil. In: BEUREN, I. M. (org.). Como elaborar trabalhos monográficos em contabilidade: teoria e prática. 2. ed. São Paulo: Atlas, 2004.

RAUPP, F. M.; BEUREN, I. M. Metodologia da pesquisa aplicável às ciências sociais. In: BEUREN, I. M. (org.). Como elaborar trabalhos monográficos em contabilidade: teoria e prática. 2. ed. São Paulo: Atlas, 2004.

RICHARDSON, R. J. et al. Pesquisa social: métodos e técnicas. 3. ed. São Paulo: Atlas, 1999.

STEVENS, G. C. Successful supply chain management. Management Decision, v. 28, n. 8, p. 25-30, 1990.

STOCK, J. R.; BOYER, S. L. Developing a consensus definition of a supply chain management: A qualitative study. International Journal of Physical Distribution \& Logistics Management, v. 39, n. 8, p. 690-711, 2009.

STOCK, G. N.; GREIS, N. P.; KASARDA, J D. Logistics, strategy and structure: A conceptual framework. International Journal of Operations and Production Management. v. 18, n. 1, p. 37-52, 1998.

Enterprise logistics and supply chain structure: the role of fit. Journal of Operations Management. v. 18, n. 5, p. 531-547, 2000.
SUKATI, I. The Study of Supply Chain Management Strategy and Practices on Supply Chain Performance. Procedia - Social and Behavioral Sciences, v. 40, p. 225-233, 2012.

TRIVINOS, A. N. S. Introdução à pesquisa em ciências sociais: a pesquisa qualitativa em educação. São Paulo: Atlas, 1987.

TURNER, J. R. Integrated supply chain management: what's wrong with this picture? Industrial Engineering, v. 25, n. 12, p. 52-55, 1993.

VAN DER VAART, T.; VAN DONK, D. P. "Buyerfocused operations as a supply chain strategy". International Journal of Operations \& Production Management, v. 26, n. 1, p. 8-23, 2006.

VENTURA, L. C.; MELO, M. C. S. Caracterização produtiva e comercial do setor de Tecnologia de Informação e Comunicação no Brasil entre os anos de 1996 a 2007. Confict, 2013.

WAGNER, M. B; MOTTA, V. T.; DORNELLES, C. SPSS passo a passo: statistical package for the social sciences. Caxias do Sul: Educs, 2004.

WISNER, J. D. A structural equation model of supply chain management strategies and firm performance. Journal of Business Logistics, v. 24, n. 1, p. 1-26, 2003.

YIN, R. K. Case study research: design and methods. 2 ed. Thousand Oaks: SAGE publications, 1994.

Data de recebimento: 10/05/14

Data de aprovação: 29/10/14 


\section{SOBRE OS AUTORES}

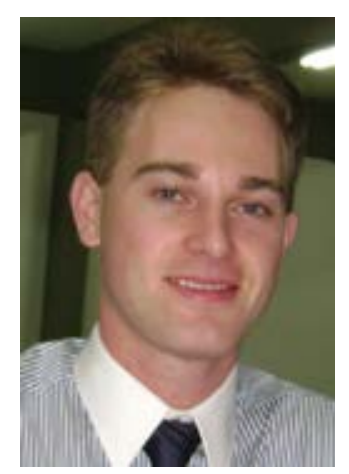

Alexandre Luis Prim

É mestrando em

Administração pela

Universidade regional de

Blumenau (FURB), especialista em Gestão de projetos pela Faculdade de Administração e

Economia (FAE) de Blumenau e tecnólogo em Produção do Vestuário graduado pelo Serviço Nacional de Aprendizagem Industrial (SENAI) deBlumenau.Atuou em indústrias de transformação de grande porte no segmento têxtil e vestuário. Foi docente do curso de Tecnologia em Produção Têxtil e Tecnologia em Produção do Vestuário do SENAI Blumenau.Atualmente, é consultor na mesma instituição, atuando nas áreas industrial-administrativas, com ênfase nos segmentos têxtil e vestuário.

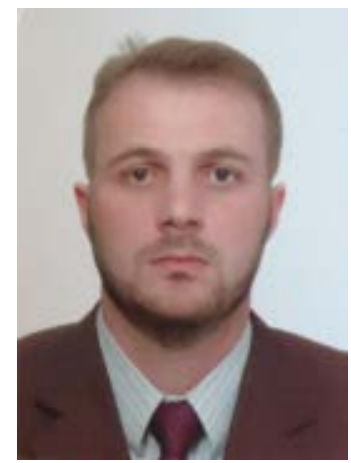

Jéferson Deleon

\section{Fávero}

É mestrando em Administração na Universidade Regional de Blumenau-FURB, especialista em Gestão Financeira e Custos pelo Instituto Catarinense de Pós-Graduação (ICPG) e administrador graduado pelo Centro Universitário Leonardo DaVinci-Uniasselvi.Atuou como professor no curso técnico em logística no SENAI - Blumenau - SC. Atuou como policial militar no estado de Santa Catarina no setor de logística bélica na cidade de Blumenau. Foi docente do curso de Administração na Cetelbras Educacional e, atualmente,écoordenador de modalidade no SENAI de Indaial - SC. 